
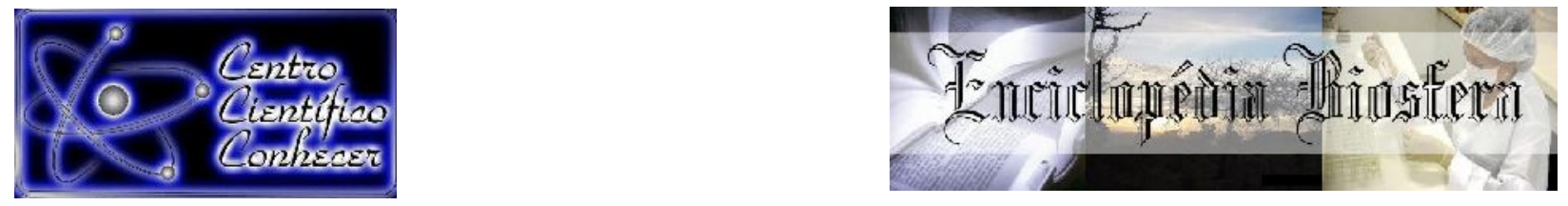

\title{
OVOS DE PARASITOS EM PORTAS DE BANHEIROS DE PONTOS TURÍSTICOS DO MUNICÍPIO DE CAROLINA, MA
}

Joana Andressa Pinheiro Rodrigues ${ }^{1}$; Tiago Paixão Ribeiro de Sousa ${ }^{1}$; Matheus Luiggi Freitas Barbosa ${ }^{1}$; Fernanda Samara Barbosa Rocha ${ }^{2}$; Luanna Soares de Melo Evangelista $^{3}$

${ }^{1}$ Acadêmicos de Medicina Veterinária, Centro de Ciências Agrárias, Universidade Federal do Piauí

${ }^{2}$ Pós-graduanda em Ciência Animal, Centro de Ciências Agrárias, Universidade

Federal do Piauí

${ }^{3}$ Prof $^{\mathrm{a}}$ - Dr- ${ }^{\mathrm{a}}$ Departamento de Parasitologia e Microbiologia, Centro de Ciências da Saúde, Universidade Federal do Piauí (luannaufpi@gmail.com)

Recebido em: 22/09/2018 - Aprovado em: 23/11/2018 - Publicado em: 03/12/2018 DOI: 10.18677/EnciBio_2018B63

Infecções por parasitos apresentam altas taxas de prevalência, principalmente em localidades onde as condições sanitárias e de higiene são precárias, e este presente trabalho avaliou a presença de ovos de helmintos em portas de banheiros de estabelecimentos turísticos do município de Carolina, Maranhão. Os banheiros foram escolhidos aleatoriamente, entre masculinos, femininos e unissex e em todos foi empregado o método da fita adesiva modificado para superfície de objetos. As fitas foram colocadas nas maçanetas das portas, em seguida fixadas em lâminas de vidro, devidamente identificadas e levadas para o Laboratório de Parasitologia do Departamento de Parasitologia e Microbiologia da Universidade Federal do Piauí. A leitura das lâminas foi realizada por microscopia óptica nas objetivas de 10x e 40x. Os resultados revelaram que das 30 lâminas analisadas, 02 (6,7\%) estavam parasitadas por ovos de Ascaris lumbricoides, sendo provenientes de banheiros unissex. Conclui-se que a presença de ovos de helmintos nestes ambientes revela que a falta de higiene dos usuários após o uso dos sanitários bem como uma limpeza inadequada destes estabelecimentos podem servir de fonte de contaminação de ascaridíase para a população local e turista.

PALAVRAS-CHAVE: fita adesiva, helmintos, higiene, sanitário.

\section{PARASITES EGGS IN BATHROOM DOORS OF TOURIST POINTS OF CAROLINA MUNICIPALITY, MA}

\begin{abstract}
Parasitic infections present high prevalence rates, especially in places where sanitary and hygiene conditions are precarious and the present study evaluated the presence of helminth eggs in bathroom doors of tourist establishments in the municipality of Carolina, Maranhão. The bathrooms were chosen randomly, among men's, women's and unisex, and in all of them the modified adesive tape method to surfaces of objects was employed. The tapes were placed on the door knobs, then fixed on glass
\end{abstract}


slides, duly identified and taken to the Laboratory of Parasitology of the Department of Parasitology and Microbiology of the Federal University of Piauí. The slides were read by optical microscopy on 10x and 40x objective. The results showed that of the 30 slides analyzed, 02 (6.7\%) were parasitized by Ascaris lumbricoides eggs, coming from unisex bathrooms. It is concluded that the presence of helminth eggs in these environments reveals that the lack of hygiene of the users after the use of the toilets as well as an inadequate cleaning of these establishments can serve as a source of ascariasis contamination for the local population and tourist.

KEYWORDS: adesive tape, helminthes, hygiene, restroom.

\section{INTRODUÇÃO}

As infecções por helmintos e protozoários representam um grave problema de saúde pública no Brasil, devido ao grande número de pessoas que são infectadas por pelo menos um endoparasito, chegando a números que alcançam até $90 \%$ da população estudada (BARBOZA; CARVALHO, 2017). Prevalências de infecções por parasitoses no país têm sido descritas em diferentes populações, principalmente nas mais carentes e a transmissão dessas doenças tem como uma das fontes de infecção as mãos, devendo ser levada em consideração, especialmente em locais públicos e de grande aglomeração humana (MENDONÇA et al., 2008), podendo ser um risco para outros indivíduos por meio de contato com estas superfícies contaminadas.

Os fatores motivadores para a ampliação das infecções por endoparasitos nos indivíduos estão correlacionados diretamente aos aspectos socioeconômicos, culturais, sanitários e ambientais e, ainda, à inadequada higiene e infraestrutura de saneamento básico (CAMELLO et al., 2016). Alguns parasitos são transmitidos pela água ou alimentos contaminados e outros por meio de larvas presentes no solo (NEVES, 2012).

A ascaridíase é uma das enfermidades parasitárias intestinais mais frequentes no país, sendo possível de ser veiculada por meio de fômites (ALVES et al., 2003). A prevalência elevada dessa parasitose desencadeia, além de problemas gastrintestinais, baixo rendimento e danos a outros órgãos, causando quadros de má absorção e diarreia, além de obstrução intestinal (ESPÍNDOLA, 2014).

Estudos epidemiológicos são necessários não só para se mensurar o problema das altas taxas de morbidade associadas às parasitoses, como também para gerar dados para o planejamento de políticas públicas voltado para o controle de enfermidades (ANDRADE et al., 2010), especialmente aquelas de fácil disseminação.

Diante da importância desse quadro, o objetivo deste estudo foi analisar a ocorrência de ovos de helmintos em superfícies de portas de banheiros de pontos turísticos do município de Carolina, Maranhão.

\section{Área do Estudo}

\section{MATERIAL E MÉTODOS}

O presente trabalho foi realizado no município de Carolina, no estado do Maranhão. Segundo Correia Filho et al. (2011) e IBGE (2016), o município de Carolina possui uma população de aproximadamente 24.000 habitantes, uma densidade demográfica de quase 4 habitantes $/ \mathrm{km}^{2}$ e está localizado no sul do Maranhão, à margem do rio Tocantins. Possui clima tropical quente e seco, com temperatura mínima de $24^{\circ} \mathrm{C}$ e máxima de $28^{\circ} \mathrm{C}$.

\section{Amostras}


Este estudo foi realizado em 13 pontos turísticos do município de Carolina, Maranhão, como resorts próximos às cachoeiras, restaurantes, bares e lanchonetes da região, os mesmos foram escolhidos aleatoriamente e realizada a coleta de material das superfícies das portas de banheiros femininos, masculinos e unissex destes estabelecimentos.

Foram coletadas 30 amostras, principalmente, das maçanetas das portas destes locais. Todas as coletas foram realizadas no mês de julho de 2017, considerado período de alta temporada na região, coincidindo com o período mais quente e com as férias dos turistas, o que aumenta consideravelmente o fluxo de pessoas nesta época do ano e as análises do material coletado ocorreram em agosto de 2017.

\section{Coletas e Análises}

As coletas foram realizadas com o auxílio de equipamentos de proteção individual (EPI's), como jalecos e luvas, promovendo a segurança e evitando a possível contaminação dos participantes da pesquisa e/ou das superfícies das portas avaliadas. Alguns estabelecimentos não possuíam maçanetas nas portas ou não apresentavam portas, não sendo possível a realização do experimento nestes locais.

Para as coletas foi utilizada a técnica proposta por Graham (1941), método da fita adesiva, modificada para a superfície de objetos. Esta técnica consistiu em aposição, por algumas vezes, de uma mesma fita adesiva transparente, cortada em tamanho $4 \times 2 \mathrm{~cm}$, na superfície desejada, no caso as maçanetas das portas dos banheiros escolhidos para esta pesquisa. Logo em seguida, a fita adesiva foi fixada em lâmina de vidro, devidamente identificada pelo nome do estabelecimento e pelo tipo de banheiro (masculino, feminino ou unissex), depositada em caixas próprias para acondicionamento de lâminas de microscopia e levadas ao Laboratório de Parasitologia do Departamento de Parasitologia e Microbiologia no Centro de Ciências da Saúde da Universidade Federal do Piauí (DPM/CCS/UFPI). As análises foram realizadas utilizando-se microscopia óptica nas objetivas de 10x e 40x, para a confirmação e identificação de ovos dos helmintos, descritas como: presença do parasito e $\mathrm{n} / \%$ de amostras parasitadas.

\section{RESULTADOS E DISCUSSÃO}

Os resultados revelaram que das 30 lâminas analisadas, 02 (6,7\%) estavam parasitadas por ovos de Ascaris lumbricoides. As amostras contaminadas foram provenientes de banheiros unissex dos estabelecimentos A2 e B2 (código de identificação dos locais visitados), o primeiro de um bar e o segundo de uma lanchonete, ambos localizados em praças públicas da cidade. As demais amostras apresentaram negatividade para parasitos através do método utilizado. A figura 01 mostra a presença de ovos de Ascaris lumbricoides nas duas lâminas positivas avaliadas neste trabalho. 


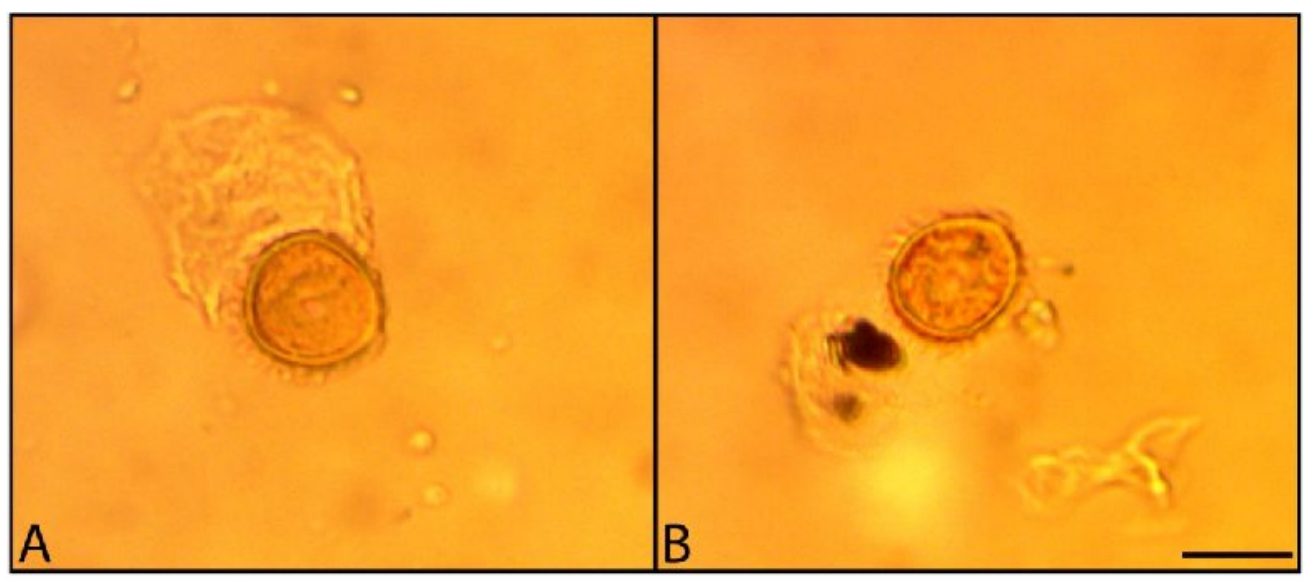

FIGURA 01 Prancha fotomicrográfica de ovos de Ascaris lumbricoides encontrados em portas de banheiros de pontos turísticos, Carolina, MA. Objetiva de 40x. Barra: 50 um. Fonte: Arquivo pessoal.

Os estabelecimentos, como restaurantes, bares e lanchonetes, eram bem próximos um do outro e segundo relatos de funcionários, geralmente são frequentados por turistas que chegam logo após a visitação das cachoeiras, sem nem passarem antes pelo hotel ou pousada para o banho. Estes aparentavam ter diferentes faixas etárias (desde crianças a idosos) e geralmente o uso dos banheiros se fazia imediatamente ao chegarem nestes locais, possivelmente trazendo patógenos via mãos e contaminando o ambiente.

Alguns trabalhos relataram que as infecções por parasitos têm relação com os padrões inadequados de higiene, sendo a habitação de ambientes públicos os locais que oferecem maiores riscos de contaminação (TOSCANI et al., 2007). Nestes casos, preconiza-se a orientação para uma limpeza mais adequada e frequente em banheiros de estabelecimentos turísticos, uma vez que recebem um grande fluxo de indivíduos durante o ano inteiro, principalmente em períodos de alta temporada.

Tais informações indicam que esses ambientes seriam então foco da contaminação, especialmente os de banheiro do tipo unissex, conforme mostra a tabela 01.

TABELA 01. Presença de ovos de Ascaris lumbricoides em banheiros de pontos turísticos do município de Carolina, MA.

\begin{tabular}{lll}
\hline Estabelecimento & Tipo de banheiro & Amostras contaminadas \\
\hline A2 & Unissex & 01 \\
B2 & Unissex & 01 \\
C2 & Unissex & 00 \\
D2 & Unissex & 00 \\
E2 & Unissex & 00 \\
F4 & Masculino & 00 \\
G4 & Feminino & 00 \\
H2 & Masculino Feminino & 00 \\
I2 & Masculino Feminino & 00 \\
J2 & Unissex & 00 \\
K2 & Unissex & 00 \\
L2 & Masculino Feminino & 00 \\
M2 & Masculino Feminino & 00 \\
\hline Total & & 02 \\
\hline
\end{tabular}


Os parasitos são veiculados por meio de água e alimentos contaminados e também pelas mãos do próprio homem, que por não ter o hábito de lavá-las antes de manipular alimentos e nem após o uso de sanitários, contribui para o processo de veiculação e transmissão destes patógenos (BELLIN; GRAZZIOTIN, 2011).

Resultados semelhantes utilizando a técnica da fita adesiva para este fim já foram relatados. Foi possível observar ovos de helmintos em superfícies de portas de banheiros de uma universidade pública de Teresina, PI (LIMA et al., 2016) e ovos de Enterobius vermicularis em salas de espera e banheiros de unidades básicas de saúde (UBS) de um município de Minas Gerais (SILVA et al., 2013), bem como em banheiros e em transportes públicos também daquele estado (BORGES et al., 2009; MURTA; MASSARA, 2009). Para estes locais, o ideal seria a utilização de medidas básicas de higiene, como limpeza e desinfecção com o uso de álcool gel como alternativas de prevenção de parasitoses.

As infecções de baixa intensidade por Ascaris lumbricoides geralmente são assintomáticas. Seus ovos possuem uma casca protetora que lhes confere maior resistência, conseguindo se manter por mais tempo no ambiente (NEVES, 2012), facilitando sua dispersão. Os vermes adultos podem causar ação espoliadora, tóxica ou mecânica nas infecções de média intensidade e nas infecções maciças, podem ocorrer, além de alterações respiratórias, o bloqueio do trânsito intestinal, ocorrendo até mesmo retardo no desenvolvimento, principalmente em crianças (SILVA et al., 2011), sendo muito importante o diagnóstico de indivíduos portadores e parasitados.

Ovos deste parasito, mesmo sendo mais pesados, podem ser facilmente disseminados no ambiente e veiculados por meio das mãos dos indivíduos parasitados, o que pode justificar a falta de higiene destes que transitaram pelos estabelecimentos pesquisados durante o período do estudo.

\section{CONCLUSÃO}

Com base nestas informações, conclui-se que a presença de ovos de helmintos nestes ambientes revela que a falta de higiene das mãos dos usuários após o uso dos sanitários, bem como uma limpeza inadequada destes estabelecimentos, servem de fonte de contaminação de ascaridíase para a população local e turista.

\section{REFERÊNCIAS}

ALVES, J. R.; MACEDO, H. W.; RAMOS Jr., A. N.; FERREIRA, L. F.; GONÇALVES, M. L. C.; ARAUJO, A. Parasitoses intestinais em região semi-árida do Nordeste do Brasil: resultados preliminares distintos das prevalências esperadas. Cadernos de Saúde Pública, v. 19, n. 2, p. 667-70, 2003. Disponível em: <http://www.scielo.br/pdf/csp/v19n2/15433.pdf>.

ANDRADE, E. C.; LEITE, I. C. G.; RODRIGUES, V. O.; CESCA, M. G. Parasitoses intestinais: Uma revisão sobre seus aspectos sociais, epidemiológicos, clínicos e terapêuticos. Revista APS, v. 13, n. 2, p. 231-240, 2010. Disponível em: <https://aps.ufjf.emnuvens.com.br/aps/article/view/736>.

BARBOZA, M. O.; CARVALHO, A. S. Análise parasitológica de contaminantes de origem fecal em banheiros femininos de uma Instituição de Ensino Superior do interior da Bahia. Revista Multidisciplinar e de Psicologia, v. 10, n. 33, p. 237-248, 2017. Disponível em: <https://idonline.emnuvens.com.br/id/article/view/616/862>. 
BELLIN, M.; GRAZZIOTIN, N. A. Prevalência de parasitos intestinais no município de Sananduva/RS, Newslab, v. 18, n. 104, p. 116-122, 2011. Disponível em: $<$ http://www.professores.uff.br/yaraadami/wpcontent/uploads/sites/155/2017/10/12Sananduva.pdf>

BORGES, C. A.; COSTA-CRUZ, J. M.; PAULA, F. M. Intestinal parasites inside public restrooms and buses from the city of Uberlândia, Minas Gerais, Brazil. Revista do Instituto de Medicina Tropical, São Paulo, v. 51, n. 4, p. 223-225, 2009.

Disponível em: <http://www.scielo.br/scielo.php?script=sci_arttext\&pid=S003646652009000400009>.

CAMELLO, J. T.; CAVAGNOLLI, N. I.; SPADA, P. K. W. D. S.; POETA, J.; RODRIGUES, A. D. Prevalence of intestinal parasites among schoolchildren and household sanitation in the urban area of Caxias do Sul, State of Rio Grande do Sul, Brazil. Scientia Medica, v. 26, n. 1, p. 1-6, 2016. Disponível em; $<$ http://revistaseletronicas.pucrs.br/scientiamedica/ojs/index.php/scientiamedica/articl e/view/21716>.

CORREIA FILHO, F. L.; GOMES, E. R.; NUNES, O. O.; LOPES FILHO, J. B. Projeto Cadastro de Fontes de Abastecimento por Água Subterrânea, estado do Maranhão: relatório diagnóstico do município de Carolina. CPRM - Serviço Geológico do Brasil, 2011.

Disponível em: <http://rigeo.cprm.gov.br/xmlui/bitstream/handle/doc/15425/relcarolina. pdf?sequence $=1>$.

ESPÍNDOLA, C. M. O. Avaliação epidemiológica das parasitoses intestinais no Parque Oswaldo Cruz, Manguinhos, Rio de Janeiro, RJ. 2014. 72 f. Dissertação (Mestrado em Medicina Tropical) - Fundação Oswaldo Cruz, Rio de Janeiro, RJ. 2014. Disponível em: <https://www.arca.fiocruz.br/handle/icict/12918>.

GRAHAM, C. F. A device for the diagnosis of Enterobius infection. The American Journal of Tropical Medicine and Hygiene, v. 21, n. 1, p. 159-161, 1941. Disponível em: <http://www.ajtmh.org/content/journals/10.4269/ajtmh.1941.s121.159>.

IBGE. Panorama das cidades. 2016. Disponível em: $<$ https://cidades.ibge.gov.br/brasil/ma/carolina/panorama $>$.

LIMA, A. D. A.; GOMES, L. R. R.; BARBOSA, M. L. F.; SOUSA, T. P. R.; MASLINKIEWICZ, A.; BACELAR, P. A. A.; FREITAS, D. R. J.; MELO EVANGELISTA, L. S. Ovos de helmintos em superfícies de banheiros de uma Universidade Pública pelo método da fita adesiva. Anais Eletrônicos da Revista Meio Norte de Medicina Laboratorial, v. 2, n. 1, p. 1-2, 2016. Disponível em: $<$ https://gpicursos.com/interagin/gestor/trabalhos/135-

968f575387338183d36b0ece46eee990.pdf>.

MENDONÇA, R. G. M.; OLIVAL, G. S.; MIMICA, L. M. J.; NAVARINI, A.; PASCHOALOTTI, M. A.; CHIEFFI, P. P. Potencial infeccioso do transporte público de passageiros da cidade de São Paulo. Arquivos Médicos da Faculdade de Ciências Médicas da Santa Casa, São Paulo, v. 53, n. 2, p. 53-57, 2008. Disponível

em: 
$<$ http://arquivosmedicos.fcmsantacasasp.edu.br/index.php/AMSCSP/article/view/393/ 446>.

MURTA, F. L.; MASSARA, C. L. Presença de ovos de helmintos intestinais em ônibus de transporte público em Belo Horizonte - Minas Gerais, Brasil. Revista de Patologia Tropical, v. 38, n. 3, p. 207-212, 2009. Disponível em: $<$ https://revistas.ufg.br/iptsp/article/view/7839/5641>.

NEVES, D. P. Parasitologia Humana. $12^{\mathrm{a}}$ ed. São Paulo: Atheneu, 2012. 546p.

SILVA, J. C.; FURTADO, L. F. V.; FERRO, T. C.; BEZERRA, K. C.; BORGES, E. P.; MELO, A. C. F. L. Parasitismo por Ascaris lumbricoides e seus aspectos epidemiológicos em crianças do Estado do Maranhão. Revista da Sociedade Brasileira de Medicina Tropical, v. 44, n. 1, p. 100-102, 2011. Disponível em: $<$ http://www.scielo.br/pdf/rsbmt/v44n1/22.pdf>.

SILVA, A. T.; MASSARA, C. L.; MURTA, F. G. L.; OLIVEIRA, A. A.; LARA-SILVA, F. $O$. Ovos de Enterobius vermicularis em salas de espera e banheiros de unidades básicas de saúde (UBS) do município de nova Serrana-MG: contribuições para o controle. Revista de Patologia Tropical, v. 42, n. 4, p. 425-433, 2013. Disponível em: <https://www.revistas.ufg.br/iptsp/article/view/27928>.

TOSCANI, N. V.; SANTOS, A. J. D. S.; SILVA, L. L. M.; TONIAL, C. T.; CHAZAN, M.; WIEBBELLING, A. M. P.; MEZZAR, A. Desenvolvimento e análise de jogo educativo para crianças visando à prevenção de doenças parasitológicas. Interface Comunicação, Saúde e Educação, v. 11, n. 22, p. 281-94, 2007. Disponível em: $<$ http://www.scielo.br/pdf/icse/v11n22/08.pdf>. 